Sinergias educativas

ISSN: 2661-6661

compasacademico@icloud.com

Grupo Compás

Ecuador

\title{
Pre-textos de una andragogía líquida. EI consumismo académico
}

Hernández de la Rosa, Eduardo

Pre-textos de una andragogía líquida. El consumismo académico

Sinergias educativas, vol. 3, núm. 2, 2018

Grupo Compás, Ecuador

Disponible en: http://www.redalyc.org/articulo.oa?id=573561678003

Esta obra está bajo una Licencia Creative Commons Atribución-NoComercial-SinDerivar 4.0 Internacional. 


\title{
Pre-textos de una andragogía líquida. El consumismo académico
}

\author{
Pre-texts of a liquid andragogy. Academic consumerism \\ Eduardo Hernández de la Rosa \\ eduardo.hernandez@soyuo.mx \\ Universidad de Oriente-Puebla, México \\ http://orcid.org/0000-0002-6333-0846
}

Sinergias educativas, vol. 3, núm. 2, 2018

Grupo Compás, Ecuador

Recepción: 15 Enero 2017

Aprobación: 25 Abril 2018

Redalyc: http://www.redalyc.org/ articulo.oa?id $=573561678003$
Resumen: El presente trabajo, aborda la tesis orientada a la reflexión en torno a la pedagogía y la andragogía como propuestas para explicar los procesos formativos en la educación superior y posgrado, se toma como referencia el contexto empírico de la ciudad de Puebla, México generando una discusión entre tres problemas andragogicos desde la posición del docente y el discente, el axiológico, epistémico y ontológico, aunado a ello se problematiza en torno a dos connotaciones del docente del contexto empírico citado, estas son el "buen docente" y el "docente bueno para" con el objeto de reflexionar sobre las exigencias que se dan en estos actores en un entorno de crecimiento institucional elevado y con exigencias orientadas hacia fines capitalistas. Finalmente se dejan nuevas reflexiones para contribuir en los pre-textos, es decir, textos no acabados sobre estos constructos, la andragogía desde el marco de reflexión del binomio docentediscente y todos los procesos formativos que implica, así como las tensiones del contexto líquido enfocadas especialmente hacia la docencia.

Palabras clave: Educación de adultos, Consumo, Docente.

Abstract: The dissertation explores the oriented to the reflection on pedagogy and andragogy as proposals to explain the formative processes in higher education and postgraduate studies, taking as reference the empirical context of the city of Puebla, Mexico generating a discussion between three andragogical problems from the position of the teacher and the student, the axiological, epistemic and ontological, coupled with this is problematized around two connotations of the teacher of the mentioned empirical context, these are the "good teacher" and "good teacher for "In order to reflect on the demands placed on these actors in an environment of high institutional growth and with demands geared towards capitalist ends. Finally, new reflections are left to contribute to the pre-texts, that is to say, unfinished texts on these constructs, andragogy from the frame of reflection of the teaching-learning binomial and all the formative processes involved, as well as the tensions of the liquid context focused especially on teaching.

Keywords: Adult education, Consumption, Teacher.

\section{INTRODUCCIÓN}

En los espacios conocidos como cotidianos, la realidad día a día nos abruma, son días emotivos, de ira, de angustia, incertidumbre, euforia, amor, tranquilidad entre otras más emociones que el ser humano se ha inventado para explicar aquello que siente, empero, el rememorar el pasado y pensar en el futuro, inmediatamente nos arranca de nuestro ser inmediato y vivido, dejándonos ausentes de un ahora que és inevitablemente y que al mismo tiempo ésta siendo, es aquí, donde la cotidianidad nos abruma, ese pesar, impulsa la no ubicación de los espacios en los que tenemos una práxis reconfigurada, tensionada y 
construida por los actores con los que se relaciona. Es ahí donde los cambios se dan de manera significativa, generando formas de abordar nuestra realidad, con todo lo que implica ello.

Es en la docencia, que las configuraciones sociales son más evidentes en tanto que se convierten en centros neurálgicos de formas de pensar, lo que hace un ambiente diverso, empero, es la docencia la que también sufre efectos de ello, así, la posición en torno a la andragogía, no busca otra cosa que hacer reflexionar sobre los efectos que tienen sobre la construcción identitaria de dos tipos de actores los "buenos docentes" y los "docentes buenos para" que corresponden a intereses de grupos específicos de la sociedad y que regularmente corresponden a intereses de orden económico. Por lo tanto, en este documento, se generan "pretextos" es decir, textos no acabados sobre la andragogía, el consumismo académico y las formas de relación que tienen estos constructos tomando como base de reflexión la realidad de la ciudad de Puebla, México, ello con el objetivo de poner en análisis, reflexión y crítica las posiciones discursivas sobre la docencia y los procesos formativos que en ella intervienen.

Finalmente, el epígrafe hace alusión a las dinámicas que promueven un reacomodo práctico e ideológico de la educación donde el consumo es un efecto tangible de lo que sucede en el quehacer cotidiano, para algunos, es parte de la premisa marxista de una "falsa conciencia" para otros, es la prueba de un sistema deficiente, lleno de vicios, otro grupo, alude que es parte de un proceso natural producto de nuestras sociedades y sus consecuentes necesidades, todo esto engloba pensar en diferentes juicios, en diferentes días, llenos de emoción categorizada como positiva o negativa, a la espera de llegar algún momento en el que se exija saber qué es lo que provoco lo vivido, en el que se busque culpables o en el que se encuentren soluciones, las emociones por el futuro son bastas, empero, el desgaste y lo que se vive ahora, arranca del imaginario de quien escribe estas líneas, un fragmento del texto del acto litúrgico católico celebrado tras el deceso de una persona, así, con la entrada de este fragmento y todo lo que podría representar esta descripción, se inician los pre-textos sobre la andragogía y el consumismo académico.

I. Argumentación de la andragogía líquida

La educación como proceso social que engloba relaciones con distintas aristas como pueden ser culturales, religiosas, cívicas, económicas y/o políticas, coinciden en la intención de formar a quienes se muestran como los sucesores en términos de cambio generacional. En esta dirección, la educación de orden formal ha acuñado de manera muy constante la connotación sobre la "pedagogía" a todo proceso formativo conducido de manera formal y sin considerar sus particularidades, empero, al retomar su origen etimológico, el constructo mantiene ciertas limitantes en la aplicación del contexto social actual, específicamente en la educación de determinadas poblaciones (2017) desde donde, las formas de relacionarnos han estado variando de manera constante por el neoliberalismo y las transformaciones que promueve, donde la educación no está exenta de ello. 
Es aquí, que el lenguaje retoma un papel importante para comprender al otro y lo otro, en términos del contenido y la significatividad que se da a los participantes y a la circunstancia en específico. Así, se hará la distinción entre pedagogía y andragogía, entendiendo que la primera se ubica específicamente hacia los actores cuyo desarrollo fisiológico esté catalogado con una conducción específica, mientras que el segundo, andragogía, trae una connotación orientada hacia las personas adultas, Kapp (1833) alude a éste, para referirse a la escuela platónica de donde lo acuña, tomándolo como una noción que engloba al aprendizaje en conjunto con lo social, identificando a la figura del docente, seguido de la auto-reflexión y la empírea. Sin embargo, es hasta 1984 cuando Knowles (1970) lo introduce desde la perspectiva de "el arte de enseñar a los adultos mayores", si bien, no se tiene una teoría construida sobre la andragogía, si se pueden enunciar puntos para el desarrollo de su comprensión

Con estos elementos, es que la propuesta de la Andragogía se ubica como un modelo más ad hoc a las necesidades de la educación superior y posgrado en donde los requerimeintos, hacen de este modelo un análisis concreto para la acción, en este sentido, es importante reconocer las diferencias de la propuesta esencial entre Pedagogía y Andragogía. De esta forma se muestran las diferencias siguiendo a Fasce (2006)

\section{Cuadro 1}

Diferencias Pedagogía y Andragogía

Cuadro 1: Diferencias Pedagogía y Andragogía
\begin{tabular}{|l|l|l|}
\hline Propuestas & Pedagogía & Andragogía \\
\hline $\begin{array}{l}\text { Propósito del } \\
\text { aprendizaje }\end{array}$ & $\begin{array}{l}\text { Se recoge la } \\
\text { informaciónque entrega } \\
\text { el profesor }\end{array}$ & $\begin{array}{l}\text { Orientado a } \\
\text { objetivos o metas } \\
\text { claramente } \\
\text { definidas }\end{array}$ \\
\hline $\begin{array}{l}\text { Rol del } \\
\text { docente }\end{array}$ & $\begin{array}{l}\text { Conducir el aprendizaje y } \\
\text { definir objetivos y } \\
\text { contenidos }\end{array}$ & $\begin{array}{l}\text { Facilitador y guia } \\
\text { según necesidades } \\
\text { de aprendices }\end{array}$ \\
\hline Experiencia & $\begin{array}{l}\text { En etapa de formación y } \\
\text { desarrollo }\end{array}$ & $\begin{array}{l}\text { Poseen experiencia } \\
\text { y necesitan } \\
\text { conectarla con lo } \\
\text { nuevo }\end{array}$ \\
\hline $\begin{array}{l}\text { Demandas de } \\
\text { aprendizaje }\end{array}$ & $\begin{array}{l}\text { Disponen de mayor } \\
\text { tiempo para dedicar } \\
\text { al aprendizaje }\end{array}$ & $\begin{array}{l}\text { Requieren } \\
\text { equilibrar sus } \\
\text { responsabilidades } \\
\text { sociales con las } \\
\text { demandas de } \\
\text { aprendizaje }\end{array}$ \\
\hline
\end{tabular}

Fuente: Obtenido de Fasce (2006)

Obtenido de Fasce (2006)

Aun cuando existen diferencias específicas sobre estos dos constructos, es importante reconocer que, pese a la reflexión que se tiene sobre la Andragogía con Kapp (1833), Merriam (1981), Knowles (1984), Norman (1999), Shannon (2003), Kaufman (2000), Adam (1970, 1984, 1987), Briseño (1993) o Briseño y Llano de la Hoz (1985), nos permite tomar una posición ontológica y sobre todo epistémica, sobre como abordamos la realidad y como se vive en ella, sin menor a dudas, la 
ubicación de este constructo puede tener limitantes, las cuales significan mirar a la Andragogía no separada de la pedagogía, sino como un modelo que busca comprender la forma en la que trasciende el aprendizaje y su construcción por quienes han dejado de ser niños en términos evolutivos, para ser considerados adultos en términos evolutivos y sociales. La pregunta específica es revisar, a la Andragogía, como una propuesta para la orientación de la docencia en la educación superior y posgrado, así como las formas en las que puede contribuir en la explicación de las prácticas actuales de la docencia en estos niveles.

Ahora bien, no se queda la reflexión aquí, los pre-textos sobre la andragogía, van enfocados y encaminados a la revisión de la aplicabilidad de contenido y significado de este constructo, pero además es expuesta a la andragogía, como una alternativa dentro de la formación de los niveles de educación superior (pregrado) y posgrado, ello, en razón a que el contexto social en el que se suscitan las reflexiones aquí presentadas emanan de lo cotidiano y por tanto de lo que la realidad -respecto a la posición del autor de este texto- expresa en los colegas académicos y que es considerado bajo la perspectiva Baumaniana de una realidad líquida.

II. El consumismo académico

$\mathrm{Al}$ momento de reflexionar sobre el espacio académico, de inmediato podríamos evocar, los seminarios y la discusión analítica, reflexiva y crítica, muy semejante a la tradición griega, de la que hemos tenido influencia, en este punto nos sumergimos en el romanticismo de lo melancólico. Empero, cuando miramos lo cotidiano desde una posición autorreflexiva, las relaciones, tensiones, angustias y necesidades de un contexto pragmático salen evidentes. El impulso de la formación en masa bajo el estandarte de la calidad educativa y la alfabetización, se difuminaron, cuando inició la sobrepoblación de los espacios universitarios, situación que iniciaría a principios de los noventa - para el caso de México-, pero tendría, un impacto tangible hasta hace algunos años. Dice Chomsky (2014) el neoliberalismo tomo por asalto a las Universidades, esto no es de extrañarse en tanto que las Universidades, son instituciones y una institución al estar integrada y dinamizada por actores concretos, sus intenciones se ven proyectadas en ella. Admitimos siguiendo al precursor de la idea moderna de Universidad, Flexner que:

(...) a university, like all other human institutions -like the church, like governments, like philanthropic organizations- is not outside, but inside the general social fabric of a given era. It is not something apart, something historic, something that yields as little as possible to forces and influences that are more or less new. It is, on the contrary -so I shall assume- an expression of the age, as well as an influence operating upon both present and future (1994: 3).

En este sentido, se identifican dos situaciones de interés para la reflexión en este texto, por un lado ¿Cómo es que la construcción ideológica orientada por el mercado ha impulsado la tendencia de la educación como un sistema de formación permanente y pragmático que dé respuesta a las necesidades del mercado laboral? Y por el otro, ¿Cómo es que la necesidad de este tipo de formación, hace que la 
práctica docente, se modifique, en tanto que la formación de adultos y por consiguiente Andragógica se convierte en un proceso masificador? Estas dos preguntas contribuyen a reflexionar, sobre las tendencias del sistema educativo del nivel superior y posgrado, puesto que ante la explosión demográfica de estudiantes cualificados, las instituciones de educación superior emergen como espacios de sobre-cualificación que permitan otorgarle "certificación" o "acreditación" a los que acuden a ella, con el objeto de tener mayores ventajas dentro de un mercado laboral saturado. Ello lleva a tendencias como lo que se denominada como "consumismo académico". Por otro lado, implica repensar, como los escenarios universitarios tanto de nivel pregrado y posgrado se convierten en puntos de reflexión sobre el proceso andragógico y la generación de nuevas alternativas para potenciar los aprendizajes en este tipo de estudiantes.

El consumismo académico como fenómeno, no solo es antagónico a los procesos sociales de la identidad "NINI", sino que además, surge como un fenómeno impulsado por quienes podrían denominarse cultura "hipster" con la necesidad de ser reconocidos socialmente por los grados académicos o estudios obtenidos, mientras que, la prolongación de estudios, cualificación, o superespecialización son aspectos que se conforman en espacios en donde la competencia en términos de mercado se vuelve cada vez menos tangible, por lo que la vinculación de formación y consumo académico, parece constituirse como un proceso de anclaje dentro de un mercado volátil y por consiguiente líquido.

El ascesis sobre el proceso de comprensión en torno a los planteamientos relacionados con el orden neoliberal, no solo alude a la construcción del consumismo académico, sino que además, implica pensar la práctica andragógica, no solo desde la perspectiva educacional y su englobe en torno a los procesos cognitivos, sino desde lo social y las necesidades del mercado, aunado a una oferta académica de bajo costo y con procesos formativos fugaces.

Es aquí, que la Andragogía no solo enfrenta el reto de la vinculación con las formas de comunicación actuales, sino, deberá estar atenta a las condiciones liquidas de aprendizaje y formación que busca la creciente población de profesionistas enfocados en incrementar su capital académico a través de credenciales. Esta situación indica que el consumismo, como categoría propia de los fenómenos expresados por el globalismo, no solo reconfiguran como es pensada la "formación" stricto sensu sino todo lo que conlleva ésta, así la profesionalización que aludía Woolgar (1991) sobre la investigación, no solo está presente en esos escenarios, sino también en la profesionalización de la andragogía, que lejos de fortalecer el perfil, lo pervierte, lo cual se traduce en la masificación del sistema de pensamiento y sobre todo, de una práctica docente, que no solo estará condicionada por los contenidos, sino también por las necesidades, para el dominio de ciertos temas relacionados con el acceso al empleo y su preservación del mismo en términos fiscales.

Luego entonces ¿Los retos de un académico tanto de nivel pregrado y posgrado, se centran únicamente en sus competencias andragógicas (ver 
Cuadro 1) La respuesta a esta pregunta puede tener dos connotaciones, por un lado, las que hacen referencia al deber ser y por otro lado, las que hoy día se necesitan tener, esta tensión dialéctica, pone un reto al académico, ontológico, en tanto que debe reflexionar si su práctica se concentra en los ideales de la educación o atiende a las necesidades del mercado y más aún, a las exigencias de su carne, en tanto que el académico actual, figura como un actor tensionado por su contexto, específicamente en el contexto de la ciudad de Puebla, México, la explosión institucional de educación superior y posgrado, ha promovido que los académicos lleven a cabo estancias fugaces de práctica docente en diversas instituciones, cuyas filosofías en algunos casos, son antagónicas, mientras que la carga administrativa puede ser igual, ello implica que el académico se encuentre en un constante dinamismo, tensionándolo sobre estos dos aspectos, el deber y el ser.

\section{MATERIALES Y MÉTODOS}

Cómo puede notarse, el problema identificado es tanto de orden ontológico, sobre una pugna entre lo que se vive y se debe hacer, pero al mismo tiempo es axiológico, en tanto que incluye, la acción como proceso de "praxis andragógica" tensionado sobre lo ético en cuanto a su posición deontológica profesional, pero también moral, en cuanto a lo que se espera que ejecute el docente; esto quiere decir, que el problema de la andragogía, no solo es de orden epistémico, sino que en él, se concentran más dimensiones, las cuales invitan a repensar a actores concretos con un cumulo de tensiones en su praxis

\section{Cuadro 1}

Competencias del Académico

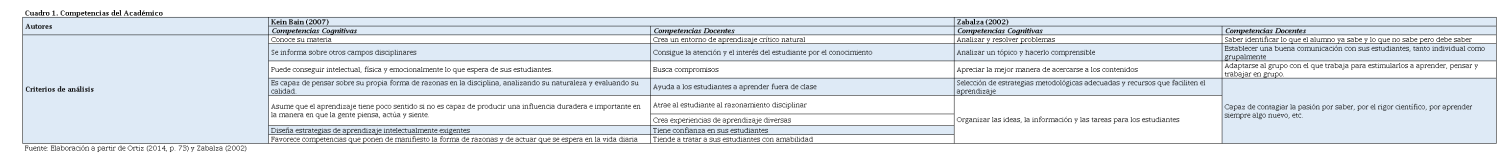

Elaboración a partir de Ortiz (2014, p. 73) y Zabalza (2002)

Con la figura 2, se busca reconocer las limitantes de la andragogía, pero al mismo tiempo, explicar cómo es que a través de ésta, es posible iniciar el reconocimiento de la realidad, una realidad que desborda los presupuestos ortodoxos de la formación educativa, en la que se reconoce la diversidad y por consiguiente las limitantes y desafíos que tienen los procesos educativos, especialmente en la andragogía y respecto a las necesidades actuales, así, el papel del docente, no solo debe contener las competencias de un "buen docente" sino, atender a las necesidades 
de su cotidianidad, lo cual, lo somete en las problemáticas expuestas epistémicas, ontológicas y axiológicas-.

Tal como se citó previamente, el contexto nacional, especialmente el de la ciudad de Puebla, tiene características que bien ejemplifican un contexto permeado por la incertidumbre y la volatilidad laboral. El docente de los niveles de posgrado y pregrado, tiene un actuar en lo líquido, al transitar en un bricolaje de instituciones sometidas a las cosificaciones normativas de cada una de estas y por consiguiente dentro de los modelos educativos o las ideologías educativas de cada institución. Ante este panorama, el reconocer la triada problemática del docente, esto es su autorreflexión en torno a cómo debe orientar su práxis y la praxis del discente (lo epistémico); en cómo debe actuar, ante instituciones que le exigen la reducción de su praxis del significado y contenido en menos tiempo y a diferentes horas, así como de instituciones que le exigen tutoría y acompañamiento a sus estudiantes, donde su ética profesional se ve tensionada y su moral parece ser moldeada entre lo que se espera del "buen docente" disciplinado y meticuloso o del "docente bueno para" aquel que sigue instrucciones, para hacer únicamente lo que se le pide (lo axiológico); y en cómo abordar la realidad, aquella que muestra incertidumbre para la subsistencia dentro de lo líquido, dentro de aquello que no es sólido, ni en su praxis, ni en el valor de la misma, donde la realidad, le exige, no ser pedagogo, en su sentido estricto, es decir, un mentor de niños, si no, ser andragogo, un facilitador de adultos, no obstante, se requiere de una andragogía líquida, aquella que se acople a todo, que se amolde a las necesidades y que deje de lado los problemas antes citados.

La ciudad de Puebla, entonces se convierte en un escenario, donde son visibles las fuerzas concéntricas del mercado, así como sus expresiones o condiciones estructurales, como lo es la incertidumbre, el conflicto social, el globalismo y el riesgo, en este sentido, pensar en el consumismo académico y la andragogía líquida, es una apuesta por mirar nuevamente a lo cotidiano, a aquello que esta frente a nosotros y que refleja como la realidad supera de forma constante nuestra capacidad de abstracción y explicación para la búsqueda de lo univoco dentro lo socialmente aceptado por un gremio de personas, para el caso aquellos que buscan los perfiles de un "buen docente" y aquellos que aluden a la educación permanente como una forma de garantizar la cualificación efectiva de los actores, cuando hay contextos, donde esta cualificación se ha sobreexplotado, dejando en tela de juicio la reflexionada e inacabada comprensión de aquello que se pretende tener como "calidad educativa".

Siguiendo los argumentos previos, el consumismo académico, puede identificarse como expresión y reproducido por la nueva racionalidad impulsada por la liquidez, donde, los mecanismos estatales han fortalecido la volatilidad laboral, el consumo de las credenciales académicas, se explica a partir de las estrategias de reproducción de Bourdieu (2011) las cuales son utilizadas para acceder a cierto capital, para el caso intelectualacadémico-laboral, que puedan ayudar a acceder a ciertas posiciones dentro de la red líquida, si bien Collins (1989) aludía a la sociedad 
credencialista, la diferencia entre ésta y el consumismo académico, estriba en que la propuesta de Collins se da en términos de la acumulación de credenciales como procesos meritocraticos y de la estratificación. Mientras que el consumismo académico, se da en términos de consumir sin siquiera necesitar los estudios o las certificaciones, esto se da en términos de la explosión institucional que brindan acceso a esto, pero además, en razón a que existe una tendencia de acceso a través de las distintas redes sociales, cuyo filtros de selección pueden ser muy escuetos o nulos.

\section{RESULTADOS}

Las reflexiones hechas hasta aquí, nos permiten describir lo siguiente, cualquier institución social esta permeada por las tensiones y desafíos del contexto social histórico y temporal concreto, eso nos invita a reconocer como es que la educación, como un ámbito social, jamás estará exento de estos retos, sino por el contrario, por lo cual, la andragogía como constructo no acabado, pero sí, como una propuesta para la reflexión, otorga pautas importantes para el análisis, reflexión y crítica de lo que acontece en el nivel superior, empero, aquí se reconoce el contexto social, como líquido siguiendo los presupuestos de Bauman (2004) sobre ello, aunado a un fenómeno producido por el neoliberalismo como lo es el consumismo académico, el cual refleja la necesidad de incrementar de manera constante el cumulo de certificaciones y acreditaciones ante un continuo bombardeo de educación.

Ahora, sobre los pre-textos que se refieren a los retos de una andragogía líquida en el posgrado, se dan en razón a que el posgrado, es el contexto educativo con mayor auge sobre el consumismo académico y donde la andragogía es más evidente en su liquidez. Así, se plantean las siguientes dimensiones como retos de la andragogía líquida en torno al posgrado frente al consumismo académico. 


\begin{tabular}{|c|c|}
\hline Dimensiones & Retos \\
\hline Epistémicos & $\begin{array}{l}\text { Académico El reto sobre lo académico se refiere } \\
\text { a como mirar al docente y al discente, en donde } \\
\text { se alude a la concepción y perfil de cada uno de } \\
\text { estos y su relación, así, el andragogo debe } \\
\text { considerar su perfil desde la perspectiva de } \\
\text { alguien que acompaña, pero que lo hace a } \\
\text { sabiendas de la existencia inminente de un } \\
\text { vertiginoso mercado, en donde su roll es } \\
\text { orientar todos los conocimientos hacia lo } \\
\text { pragmático y por consiguiente útil para el } \\
\text { discente, en este sentido, el andragogo, reconoce } \\
\text { en el discente, el papel de un actor activo, } \\
\text { consciente de su realidad, pero también } \\
\text { permeado con influencias de orden neoliberal, } \\
\text { asi el reto que se le presenta es el de evaluar } \\
\text { permanentemente su práxis andragógica sobre } \\
\text { que contenidos dirigir y cómo hacerlo, teniendo } \\
\text { en cuenta el papel sustantivo que tiene frente al } \\
\text { consumismo académico y otros fenómenos. Así } \\
\text { se tiene: } 1 \text {. Responder a las necesidades del } \\
\text { contexto. } 2 \text {. Atender las necesidades de los } \\
\text { discentes en un contexto volátil. } 3 \text {. La } \\
\text { delimitación de la concepción andragógica de su } \\
\text { praxis académica. }\end{array}$ \\
\hline Ontológicos & $\begin{array}{l}\text { Praxis La praxis del andragogo, se ve } \\
\text { tensionada por las exigencias institucionales, } \\
\text { que como ya se ha dicho, se concentra en } \\
\text { actores cuyo quehacer no tiene un punto de } \\
\text { concentración sino varios, lo que lo lleva a } \\
\text { responder a diferentes exigencias, así el } \\
\text { andragogo, debe poner en reflexión como poder } \\
\text { rendir y mantener su identidad frente a estos } \\
\text { procesos normativos de cosificación. Mientras } \\
\text { que, también están presentes las exigencias } \\
\text { personales, que tienen que ver con la } \\
\text { subsistencia en cuanto al ingreso económico y } \\
\text { la optimización de los recursos para lograr } \\
\text { mantenerse dentro de los parámetros de } \\
\text { exigencias, en cuanto a que a mayor esfuerzo, } \\
\text { mayor consumo de energía para el desarrollo de } \\
\text { las actividades, así los retos se pueden definir } \\
\text { en los siguientes puntos: 1. Hacer frente a la } \\
\text { cosificación de identidades por las } \\
\text { interdependencias normativas. } 2 \text {. Comprender } \\
\text { la praxis personal dentro de un escenario } \\
\text { laboral y responder con la optimización de } \\
\text { recursos para el desarrollo de una praxis } \\
\text { idónea. } 3 \text {. Generar procesos de organización y } \\
\text { comunicación administrativa para lograr } \\
\text { permanecer dentro de la dinámica institucional } \\
\text { y las necesidades personales. }\end{array}$ \\
\hline Axiológicos & $\begin{array}{l}\text { Autoconcepción El siguiente reto, tiene que } \\
\text { ver con la autoconcepción, la cual se da en dos } \\
\text { direcciones una de orden ética y otra de órden } \\
\text { moral, que como se ha dicho, tiene a su vez dos } \\
\text { enfoques. Así, lo deontológico (ético) forma } \\
\text { parte de comprender cómo es que la praxis } \\
\text { andragógica se da en términos del análisis de lo } \\
\text { individual y de lo que debe hacerse dentro del } \\
\text { orden disciplinar, que conlleva la definición de } \\
\text { un acontecer ético de la praxis reflexiva sobre lo } \\
\text { que uno debe hacer desde su visión disciplinar- } \\
\text { profesional. Mientras que lo moral, se da en } \\
\text { términos de lo que el actor debe ofrecer a un } \\
\text { conjunto social, que lo encajona entre lo que es } \\
\text { "bueno" para lo social, y lo que es "bueno" para } \\
\text { un grupo de lo social. Esto se concreta en los } \\
\text { retos siguientes: 1. Lo ético, como un proceso de } \\
\text { reflexión continúo sobre la praxis disciplinar, } \\
\text { que lo ubica en responder a las necesidades de } \\
\text { diferentes contextos institucionales. } 2 \text {. Lo } \\
\text { moral, como un proceso social que tensiona la } \\
\text { praxis del actor, por lo que debe responder a las } \\
\text { necesidades de lo social y lo de un grupo de lo } \\
\text { social, por lo que debe enfrentarse a tomar una } \\
\text { decisión sobre su actuar. } 3 \text {. Es necesario } \\
\text { reconsiderar el papel que juega el andragogo } \\
\text { sobre su praxis, en el que se vincula su ejercicio } \\
\text { profesional y las necesidades sociales, por lo } \\
\text { que se vuelve prioritario mantener una posición } \\
\text { que garantice una visión axiológica integral } \\
\text { sobre la praxis andragógica. }\end{array}$ \\
\hline
\end{tabular}

Fuente: Elaboración propia

Elaboración propia 
Hasta aquí, los retos que se han descritos, nos permiten ubicar que la andragogía líquida como propuesta de acción frente al consumismo académico en el posgrado, dicha propuesta, se plantea con sus limitantes y bondades, partiendo de la premisa, de responder al contexto líquido.

\section{DISCUSIÓN}

A lo largo del texto, la tesis que se buscó exponer se centra en mirar a la andragogía líquida como una propuesta de ubicación sobre los actores que ejercen su práxis docente en el nivel pregrado y posgrado, específicamente, en el posgrado, la andragogía líquida se expone como un quehacer que intenta explicar escenarios vertiginosos, para el caso concreto el de la ciudad de Puebla, México en la que la explosión institucional ha promovido una movilidad constante de académicos, empero, esta movilidad trae otros problemas situados sobre la concepción de la práctica y por consiguiente los elementos epistémicos, ontológicos y axiológicos, de los cuales se evidencian los retos, en términos de una andragogía líquida que responda como un frente de acción sobre el consumismo académico, el cual, no solo muestra un fenómeno social, sino además evidencia la cosificación de identidades y los retos a los que se enfrentan los actores, específicamente aquí, el de los andragogos.

Surgen entonces nuevas inquietudes bajo las mismas orientaciones, epistémicas, ontológicas y axiológicas, estas son, ¿Cuál es el perfil que discursivamente se maneja en el escenario formal de las instituciones? ¿Cuáles son las "competencias", "facultades" que un académico de educación superior y posgrado debe tener? ¿Cuáles son las "Competencias", "Facultades" que un académico de educación superior y posgrado necesita fortalecer para responder al contexto? ¿Qué es lo que espera el sistema educativo de educación superior y posgrado de los estudiantes, de aquellos que se someten a estos estudios? Específicamente ¿Qué se espera de los estudiantes de posgrado que egresando, no cuentan con opciones laborales, aun cuando tienen estudios de posgrado acreditados bajo el eslogan de "calidad"? su futuro es ¿Convertirse en un académico tensionado por la docencia y la explosión institucional masificador? ¿Son estas dinámicas efectos del profesionalismo, o solo un efecto de una masificación que busca la sobrecualificación enmascarada de profesionalismo? Con estas re-flexiones iniciamos nuevos pre-textos para el análisis, reflexión y crítica de nuestro quehacer cotidiano.

\section{Referencias}

Adam, F. (1970). Andragogía: ciencia de la educación de adultos. Caracas: Federación Interamericana de Educación de Adultos (FIDEA).

Adam, F. (1984). Universidad y educación de adultos. En Siete visiones de la educación de adultos. Pátzcuaro, México: CREFAL.

Adam, F. (1987). Andragogía y docencia universitaria. Caracas: Federación Interamericana de Educación de Adultos (FIDEA). 
Bain, K. (2007). Lo que hacen los mejores profesores de universidad. Valencia, España: Universitat de Valéncia.

Bauman, Z. (2004). La sociedad sitiada. Buenos Aires, Argentina: Fondo de Cultura Económica

Bourdieu, P. (2011). Las estrategias de reproducción social. Argentina: Siglo XXI Editores S. A.

Briceño M. y Llano De la Hoz. (1985). Discrepancias y relaciones entre Andragogía y Pedagogía. Rev. Educación y Ciencias Humanas. UNESR. (4).

Briceño, M. (1993). La Investigación Acción y el Androgogo.PLANIUC. 12, (20), 215-220

Chomsky, N. (2014). El neoliberalismo tomó por asalto a las Universidades. El espectador. Recuperado de http://www.elespectador.com/noticias/educacion/el-neoliberalismotomo-asalto-universidades-noam-chomsk-articulo- 480438

Collins, R. (1989). La sociedad credencialista. México: Akal.

Fasce H., E. (2006). Tendencias y Perspectivas Andragogía. Rev. Edu. Cienc. Salud. 3 (2), 69-70.

Flexner, A. (1994). Universities: American, English, German. New Brunswick, Estados Unidos: Transaction Publishers.

Kapp, A. (1833). Plato's Erziehungslehre, als Pädagogiik Für die Einzelnen und als Staatspädagogik. Alemania: Minden und Leipzig.

Kaufman D, Mann K and Jennett P. (2000). Teaching and learning in medical education: how theory can inform practice. Edimburgh, Escocia: ASME

Knowles M. (1970). The modern practice of adult education: andragogy versus pedagogy. New York: Association Press.

Knowles M. (1984). Andragogy in action. San Francisco, Estados Unidos: JosseyBass

Merriam S. (1981). Updating our knowledge of adult learning. J Continnum Ed in the Health Professions. Journal of Continuing education in helath professions. 16(3), 136-143

Norman G. (1999). The adult learner: a mythical species. Acad Med. 74(8), 886-9

Ortiz, S. (2014). La formación humanista en la educación superior. Modelo Humanista Integrador basado en Competencias. México: Gedisa.

Shannon S. (2003). Adult learning and medical education programmes. The Lancet, 361(9353), 266.

Woolgar, S. (1991). Ciencia: abriendo la caja negra. España: Anthropos

Zabalza, M. (2002). La enseñanza universitaria. El escenario y sus protagonistas. Madrid: Narcea. 